\title{
Accelerating Inflammation Resolution to Counteract Chemical Cutaneous Injury
}

Satyanarayana Achanta ${ }^{1 \dagger}$, Narendranath Reddy Chintagari ${ }^{2 \dagger}$, Shrilatha Balakrishna ${ }^{2 \square}$, Boyi Liu ${ }^{1 *}$, and Sven-Eric Jordt ${ }^{1,3}$

${ }^{1}$ Department of Anesthesiology, Duke University School of Medicine, Durham, NC - 27710, USA

${ }^{2}$ Department of Pharmacology, Yale University School of Medicine, New Haven, CT - 06511, USA

${ }^{3}$ Pharmacology and Cancer Biology, Duke University School of Medicine, Durham, NC - 27710, USA

$\dagger$ contributed equally

Current affiliations:

*Department of Neurobiology and Acupuncture Research, The Third Clinical Medical College, Zhejiang Chinese Medical University, Hangzhou, China

$\square$ Office of Animal Research Support, Yale University, 25 Science Park, New Haven, CT, USA

Corresponding author:

Sven-Eric Jordt, $\mathrm{PhD}$

Box 3094, MS27, Durham, NC 27710-3094

Phone: +1919684 1327

Fax: +19196842411

Email: sven.jordt@duke.edu

Running title: Lipid mediators mitigate chemical skin injury 


\section{Abbreviations:}

RvD1 (Resolvin D1); RvD2 (Resolvin D2); CS (2-Chlorobenzalmalononitrile); CEES (2-chloroethylethyl-sulfide); SM (sulfur mustard); HD (sulfur mustard); MMP-9 (matrix metallo protein-9), IL-1 $\beta$ (Interleukin-1 beta), KC/CXCL1 (keratinocyte chemoattractant)/(chemokine (C-X-C motif) ligand 1); CXCL2 (chemokine (C-X-C motif) ligand 2); IL-6 (interleukin-6); ELISA (enzyme linked immune sorbent assay); PBS (phosphate buffer saline); MEVM (mouse ear vesicant model); i.p (intraperitoneal); i.v (intravenous); NSAIDs (nonsteroidal anti-inflammatory drugs) 


\begin{abstract}
Chemical exposure to vesicants such as sulfur mustard (SM), and electrophilic riot control agents such as 2-chlorobenzalmalononitrile (CS) tear gas agent, cause strong cutaneous inflammation. Classical antiinflammatory treatments have focused on interference with target initiation and maintenance of inflammation, with mixed outcomes. Inflammation is broadly classified into three temporal phases, initiation, amplification and maintenance, and resolution. Resolution of inflammation was thought to be a passive process but the recent body of literature shows that resolution is an active process and is mediated by fatty acid-derived mediators (specialized pro-resolving mediators, SPMs). We hypothesized that accelerating resolution phase of inflammation may attenuate the exaggerated inflammatory response following chemical threat exposure, leading to decreased morbidity and improved recovery. In this study, SPMs, such as Resolvin D1 (RvD1) and Resolvin D2 (RvD2), were administered to mice at nanogram doses post-exposure to an SM analog, 2-chloroethyl-ethyl-sulfide (CEES) or CS tear gas agent. SPMs decreased edema (ear thickness and punch biopsy weights), pro-inflammatory cytokines (IL-1 $\beta$, CXCL1/KC, MIP2) and protease marker (MMP-9), and vascular leakage (determined by IRDye 800 CW PEG) while improving histopathology in cutaneous chemical injury mouse models. These results support our hypothesis and pave the way for SPMs for further development as potential medical countermeasures for chemical threat agents-induced skin injuries.
\end{abstract}

Keywords: specialized pro-resolving mediators (SPMs), CS tear gas agent, 2-Chloroethyl-ethyl-sulfide (CEES), cutaneous inflammation, IRDye 800 CW PEG, sulfur mustard 


\section{Introduction}

\section{Chemical threat agents-induced skin injuries:}

The treatment of injuries caused by exposures to chemical warfare agents or hazardous industrial chemicals remains challenging due to limited information about the injury mechanisms and biological targets. Exposures to chemical threat agents oftentimes induce strong inflammatory tissue responses that contribute to morbidity and delayed tissue repair and recovery. Skin blistering agents (vesicants) such as sulfur mustard (HD or SM) in mustard gas, or its analog, 2-chloroethyl-ethyl-sulfide (CEES), while not immediately irritating, induce delayed inflammatory responses that contribute to the separation of skin layers and blister formation hours after exposure (1-6). This response is initiated by the alkylating activity of HD and CEES, followed by induction of oxidative stress and the formation of lipid peroxidation products. Within a short time, pro-inflammatory cytokines and chemokines can be detected, including IL- $1 \alpha / \beta$, IL-6, IL-8, TNF- $\alpha$, GM-CSF and KC, and inflammatory cells infiltrate the exposed skin, including neutrophils, mast cells, and macrophages. Proteases such as MMP-9 are induced that contribute to edema formation, tissue destruction and blistering (6). Sulfur mustard wound often shows incomplete healing, with patients burdened by chronic skin inflammation and pruritus even decades after exposure $(2,7)$.

Other chemical agents cause more immediate harm, followed by an exaggerated inflammatory response that aggravates tissue injury and delays recovery. For example, skin and eye exposures to tear gas agents can cause immediate chemical burns and irritation due to their high chemical reactivity leading to the rapid modification of proteins, lipids and other biomolecules (8). Skin exposure to tear gas agents such as 2-chlorobenzalmalononitrile (CS tear gas agent) cause pain and irritation, and, depending on the exposure concentration and site, can lead to burns, subcutaneous edema and bulla formation (811). Some studies have reported contact dermatitis following repeated exposure to CS tear gas agent (12-14). Several short- and long-term effects of exposure to CS tear gas agent were reported (14-16).

While inflammation is essential for host defense and may prevent infiltration of the chemical wound by pathogens and contribute to clearance of debris, experiments in chemically exposed neutrophil-depleted animals demonstrated that these pro-inflammatory cells exacerbate the injury and contribute to morbidity (17). Taken together, these reports support the idea that exaggerated inflammation is a major driver of progressive tissue injury hours (and even days) after the chemical threat exposure has ended.

Several treatment options, including the use of nonsteroidal anti-inflammatory drugs (NSAIDs), are published for HD and CS tear gas-induced cutaneous injury, with mixed outcomes $(8,18,19)$. 
Despite their use as chemical warfare agents more than a century, there is no specific antidote for sulfur mustard or tear gas agent-induced cutaneous injuries (9). Although some therapeutic agents were tested for SM and CS tear agent-induced injuries, symptomatic treatment is the general line of treatment $(8,9$, 18, 20-23). Decontamination of affected subjects is crucial in mitigating the injuries. However, decontamination of contaminated surfaces is also difficult as CS tear gas agent is almost insoluble in water and only slightly soluble in ethyl alcohol and carbon tetrachloride. Similarly, the presence of sulfur mustard in different physical states complicates decontamination. The actual concentrations of incapacitating agents exposed to civilians and law enforcement authorities are highly variable, which further complicates the triaging of clinical symptoms and medical management. While some of the potential therapeutic drug candidates are being tested in pre-clinical studies and are promising, none of the compounds were approved by US FDA $(6,8,18,19,24-28)$. The lack of effective antidotes for SM and CS tear gas-induced skin injuries constitute the need for continued screening and development efforts.

Resolution of inflammation: An active mechanism driven by newly discovered omega 3-fatty acidderived mediators

The inflammatory response is divided into three temporal phases, initiation, amplification and maintenance, and resolution. Classical anti-inflammatory treatments have focused on interference with target pathways involved in the initiation and maintenance of inflammation. These strategies have only been partially successful, due to the large variety of pathways and pathologies involved. Moreover, selective and non-selective cyclooxygenase-2 (COX2) inhibitors show beneficial therapeutic effects but with obvious side effects. For many inflammatory conditions with symptoms resembling the outcomes of chemical threat injuries (such as asthma, ARDS, or dermatitis), clinical trials using cytokine inhibitors, steroids or NSAIDs have resulted in mixed outcomes, suggesting continued demand for the development of new clinical strategies counteracting inflammation.

A new area of inflammation research has focused on the process of inflammation resolution (2932). Resolution of inflammation was thought to occur due to the lack of inflammatory drive when concentrations of inflammation-initiating and -maintaining mediators are removed or in decline. However, new studies show that the resolution of inflammation is an active mechanism involving the activation of signaling pathways during inflammation initiation, and the later generation of fatty-acidderived specialized pro-resolving mediators (SPMs) that activate resolution mechanisms. These mediators include resolvins, lipoxins, protectins, and maresins. Resolvins and protectins are omega-3 fatty acids derived from docosahexaenoic acid (DHA; C22:6) or eicosapentaenoic acid (EPA; C20:5), 
the two dietary fatty acids widely consumed due to their reported anti-inflammatory and other beneficial effects (29). These mediators are produced enzymatically in human blood by neutrophils, with increased concentrations observed following aspirin treatment (33). SPMs are shown to bind to a range of Gprotein-coupled receptors and ion channels such as transient receptor potential (TRP) ion channels to mediate their pro-resolving effects $(29,34,35)$. In general, SPMs help injured tissues to return to their original architecture and function by promoting resolution through recruitment of non-inflammatory monocytes. Then, cellular debris, inflammation causative agent(s) and excess neutrophils will be removed by macrophages $(30,32,36,37)$.

Therapeutic potential and clinical development of pro-resolving mediators

SPMs, when administered exogenously, have shown potent anti-inflammatory effects in a wide range of animal disease models, including lung injury, pain, dermatitis, arthritis, and colitis (32, 33, 35, 38-47). The development of SPMs as therapeutics is an active area of pharmaceutical research, with some SPMs such as Resolvin E1 and Resolvin D1 are currently in tests in clinical trials for the treatment of inflammatory eye conditions, psoriasis, chronic pain, and many others (https://clinicaltrials.gov/ct2/results?cond=\&term=specialized+pro$\underline{\text { resolving }+ \text { mediators }+\mathrm{OR}+\mathrm{RvD} 1+\mathrm{OR}+\mathrm{RvD} 2+\mathrm{OR}+\mathrm{RvE} 1+\mathrm{OR}+\mathrm{MaR} 1 \& \text { cntry }=\& \text { state }=\& \mathrm{city}=\& \text { dist }=\& \mathrm{Se}}$ arch=Search, accessed 02/05/2019). In addition to bio-identical agents, chemically modified derivatives have been synthesized with improved stability and specificity $(32,36)$.

We hypothesize that accelerating resolution phase of inflammation may attenuate the exaggerated cutaneous inflammatory response following chemical threat agent exposure to sulfur mustard gas and tear gas agent, leading to decreased morbidity and improved recovery. Several new families of lipid-derived, local acting chemical mediators, which are derivatives of omega-3 fatty acids that enhance the resolution of the inflammatory process and restore the original architecture of injured tissues have been identified. The objective of these studies was to test the therapeutic potential of SPMs in mouse models of CS tear gas agent- and CEES-induced cutaneous injury.

\section{Materials and methods}

Specialized pro-resolving mediators (Resolvin D1 (RvD1) and Resolvin D2 (RvD2)) were purchased from Cayman chemicals Inc., Ann Arbor, MI, USA. IRDye $800 \mathrm{CW}$ was purchased from LiCor, Lincoln, NE, USA. 2-chloroethyl-ethyl-sulfide (CEES), a sulfur mustard analog was purchased from Sigma-Aldrich, MO, USA. CS tear gas agent was purchased from Combi-Blocks, San Diego, CA. 
All other chemicals and reagents used were obtained from scientific suppliers, such as Fisher Scientific and Sigma Aldrich.

C57BL/6 mice (male, 8 weeks) were purchased from Charles River, CT. Mice were housed in Association for Assessment and Accreditation of Laboratory Animal Care (AAALAC) International certified facilities. All animal protocols were approved by the Institutional Animal Care and Use Committee (IACUC), Yale University, New Haven, CT and Duke University School of Medicine, Durham, NC. Mice were given access to ad libitum mouse chow and water. Mice were given at least 48 hr acclimation period before initiating studies. In adherence to the Office of Animal Welfare Assurance guidelines, cage enrichment was done when mice were housed alone transiently.

\section{2-chloroethyl-ethyl-sulfide mouse ear vesicant model (CEES MEVM)}

Sulfur mustard analog-induced mouse ear vesicant model was developed as described in Achanta et al., 2018 (6). Briefly, the right ears of male 8 week old C57BL/6 mice were exposed to a total single dose of $0.2 \mathrm{mg}$ (in $20 \mu \mathrm{L}$, ten microliters on each side of right ear) of 2-chloroethyl-ethyl-sulfide (CEES), a sulfur mustard analog following sevoflurane anesthesia. The left ears were exposed to vehicle (dichloromethane) that served as controls. One hour later, mice were administered a single dose of SPM (RvD1 or RvD2) at $2 \mu \mathrm{g} / \mathrm{kg}$ intraperitoneally (i.p), in PBS with $0.1 \%$ ethanol (injection volume: 10 $\mathrm{mL} / \mathrm{kg}$ body weight). Control animals received vehicle (PBS with $0.1 \%$ ethanol) only. Figure $1 \mathrm{~A}$ depicts the CEES MEVM study paradigm and dosage regimen. We prepared fresh SPM solutions within 30 minutes before injection. Briefly, SPMs were evaporated under a gentle stream of nitrogen gas and reconstituted in $0.1 \%$ ethanol in PBS.

\section{Visualizing extravasation of inflammatory exudate}

To visualize the extravasation of inflammatory exudate into surrounding tissues and the healing process in CEES exposed ears, we injected IRDye 800CW intravenously at $18 \mathrm{hr}$ post-CEES exposure under a brief sevoflurane anesthesia. We scanned anesthetized mice (ketamine and xylazine at 80-120 and 5-10 mg/kg body weight i.p., respectively) in dorsal recumbency at $23 \mathrm{hr}$ post-CEES exposure using Li-Cor Odyssey CLX $^{\circledR}$ fitted with an accessory, Mouse-Pod ${ }^{\circledR}$ (Li-Cor, Nebraska, USA). Mice were maintained at $37{ }^{\circ} \mathrm{C}$ while under anesthesia and during the scanning procedure. We used the following settings for scanning of mice - channels: 700/800; scan resolution: $42 \mu \mathrm{m}$, intensities: 1 for both channels; data analysis: small animal; scan quality: medium (increasing the quality of scan will increase the time of scanning); focal offset: $1 \mathrm{~mm}$. Data were analyzed using the Small Animal Image Analysis Suite provided by the manufacturer. 
Ear punch biopsy sample collection, pro-inflammatory cytokine, and histopathology analysis

Mice were euthanized $24 \mathrm{hr}$ post-CEES exposure in the $\mathrm{CO}_{2}$ chamber and then by a secondary method, approved by the IACUC and AVMA guidelines for euthanasia. Ear thickness was measured using spring-loaded electronic calipers (Mitutoyo QUICKmini, Japan). Three $4 \mathrm{~mm}$ ear punch biopsies were excised (4 mm Biopsy Punch, Miltex Inc., York, PA, USA) and weighed to determine edema as described previously (6). One punch biopsy was used to determine concentrations of pro-inflammatory mediators known to contribute to the vesicant injury. Briefly, ear punch biopsy samples were homogenized in lysis buffer $(50 \mathrm{mM}$ Tris-base, $150 \mathrm{mM} \mathrm{NaCl}, 5 \mathrm{mM}$ EGTA supplemented with EDTAfree complete protease inhibitor (Roche Diagnostics GmbH, Mannheim, Germany) and 0.5\% Triton X100), using a Bullet Blender and Zirconium Oxide Beads (NextAdvance ${ }^{\circledR}$, Averill Park, NY) (6). Using the enzyme-linked immunosorbent assay (ELISA), we examined the concentrations of matrix metalloproteinase 9 (MMP-9), IL-1 $\beta \quad$ (interleukin-1 beta), KC/CXCL1 (keratinocyte chemoattractant)/(chemokine(C-K-X motif) ligand 1), MIP-2/CXCL2 (macrophage inflammatory protein 2)/(chemokine(C-K-X motif) ligand 2), and interleukin-6 (IL-6) in homogenized supernatant protein extracts of ear punch biopsy samples. R\&D Systems cytokine kits (Minneapolis, MN) or a high throughput multiplex cytokine assay system (Milliplex MAP Mouse Cytokine/Chemokine Magnetic Bead Panel, Millipore, MO, USA) was used for cytokine quantification, following manufacturer's instructions. All samples were analyzed in at least duplicate on Infinite M200 Pro (Tecan, Germany) or Bio-Plex 200 system (Bio-Rad, Hercules, CA). The concentrations of cytokines were quantified using a standard curve or a 5-parameter logistic regression analysis. Concentrations of samples outside the range of standard curves were excluded. The concentrations of protein in homogenate samples were determined using Pierce BCA protein assay (Thermo Scientific, Rockford, IL).

One punch biopsy was fixed in $10 \%$ formaldehyde, embedded in paraffin, sectioned at $5 \mu \mathrm{m}$ thickness, and stained with hematoxylin and eosin $(\mathrm{H} \& \mathrm{E})$ as per standard protocols. Images were obtained with a Zeiss Axio Imager Z1 microscope and analyzed by AxioVision Rel. 4.7 software (Zeiss, Munich, Germany). Cutaneous histopathology was assessed based on guidelines in Silny et al., 2005 (6, 48).

\section{Electrophilic agent (CS tear gas agent) induced cutaneous inflammation:}

Mice were briefly anesthetized with sevoflurane. We exposed right ears of mice with $20 \mu \mathrm{L}$ (10 $\mu \mathrm{L}$ on each surface) of $200 \mathrm{mM}$ CS tear gas agent and applied an equal volume of dimethyl sulfoxide (DMSO, a vehicle for CS tear gas agent application) on left ears. We administered either SPM (RvD1 or RvD2) at $5 \mu \mathrm{g} / \mathrm{kg}$ body weight i.p or vehicle (PBS with $0.1 \%$ ethanol) at 30 minutes and 4 hours post- 
CS exposure. We euthanized mice at 6.5 hours post-CS exposure. Ear thicknesses, ear punch biopsy weights; tissue homogenization to harvest protein extracts, cytokine analysis, and H\&E staining were done as described in the above CEES MEVM sections. Figure 5A depicts the study paradigm and dosing regimen.

\section{Data Analysis and Statistics:}

Data were analyzed using GraphPad Prism 7 for Windows, GraphPad Software, La Jolla, CA, USA. Statistical difference was tested either by an unpaired two-tailed t-test or by one-way ANOVA with Tukey's Multiple Comparison Test. Error bars are represented as the standard error of mean estimate (SEM). Statistical significance was denoted by $* \mathrm{p}<0.05$ or $* * \mathrm{p}<0.01$ or $* * * \mathrm{p}<0.001$.

\section{Results}

\section{CEES MEVM}

Cutaneous exposure to CEES resulted in tissue edema and microvesicles. Ear thickness, measured with spring-loaded calipers, increased by $49.4 \pm 21 \%$ compared to vehicle (dichloromethane) exposed ears. Ear punch biopsy weights, measured from three 4-mm punch biopsies, increased by $57.5 \pm 23 \%$ compared to vehicle exposed ears. Treatment of mice with RvD1 or RvD2 post-CEES exposure decreased tissue edema, measured with ear thickness and ear punch biopsy weights (Figures $1 \mathrm{~B}$ and 3A). Profound vascular leakage was noted in ears exposed to CEES due to vascular dilation, increased diapedesis, and accumulation of inflammatory exudate in the subcutaneous tissue. Whereas, vascular leakage was significantly decreased in mice that were treated with RvD1 or RvD2 post-CEES exposure (Figures 2A-B and 4A-B). The intensity of IRDye $800 \mathrm{CW}$ in SPM-treated groups was significantly lower than the vehicle-treated animals. Inflammatory cytokine markers, such as MMP-9, IL-1 $\beta$, CXCL1/KC, CXCL2/MIP2, and IL-6, were decreased in treatment groups compared to control (vehicle) mice, suggesting that acceleration of inflammation resolution resulted in diminished tissue destruction by proteases and recruitment of inflammatory neutrophils (Figures 2C and 4C). Tissue biopsies of CEES exposed mice treated with SPMs showed dramatically attenuated edema and microvesicle formation (separation of epidermal and dermal layers) and reduced inflammatory infiltrating cells, resulting in almost normal ear thickness (Figures $1 \mathrm{C}$ and 3B).

\section{CS tear gas}


Exposure to CS tear gas agent-induced acute inflammation, evidenced by erythema and edema within few minutes after exposure. Compared to the vehicle (DMSO-exposed ears), ear thickness and ear punch biopsy weights increased by $63 \pm 21 \%$ and $75 \pm 23 \%$, respectively.

Post-exposure treatment of CS tear gas agent-induced acute cutaneous inflammation with SPMs (RvD1 or RvD2) decreased tissue edema compared to the control group (Figure 5B and 6A). SPMs decreased pro-inflammatory cytokines (IL-1 $\beta$, CXCL1/KC, CXCL2/MIP2, and IL-6) and MMP-9 (Figures 3B and 4B). In tissue histopathology sections of CS tear exposed ears, edema was an obvious finding in addition to infiltration of cells. In treated groups, edema, infiltration of pro-inflammatory cells, and keratinolysis were decreased compared to control groups (Figure 3C and 4C).

\section{Discussion}

Skin is the body's largest organ system with an average body surface area ranging from $1.6-1.9$ $\mathrm{m}^{2}$ in humans. Therefore, cutaneous injuries following chemical exposure are the most common sequelae. In this study, skin injury caused by sulfur mustard analog, CEES and CS tear agent were studied in mice; and the therapeutic potential of SPMs was investigated in chemical threat agent-induced cutaneous injuries. To the best of authors' knowledge, this is the first report on the use of SPMs as therapeutic agents in chemical agent-induced cutaneous inflammation or in any other medical countermeasure studies.

\section{CEES MEVM}

Sulfur mustard interacts with biological matrices in a wide variety of cascade of pathophysiological events and therefore, difficult to control inflammation. Although the toxicological effects of sulfur mustard on the ocular and respiratory system are widely studied, information for cutaneous effects is scarce. CEES is commonly used as an analog to study the effects of sulfur mustard (HD). CEES MEVM in this study recapitulated previously published reports elsewhere $(5,6)$. Edema or erythema of exposed ears was not seen immediately. CEES is a mono-functional alkylating analog of sulfur mustard which forms adducts with the biological interface, unlike sulfur mustard. CEES is 100 times less potent than sulfur mustard. Visible blisters/vesicles were not seen in CEES MEVM, unlike sulfur mustard. However, CEES is commonly used as a surrogate agent to study the effects of sulfur mustard under laboratory settings due to its similarities of chemical and toxicological effects to sulfur mustard. 
In this study, several pro-inflammatory cytokines markers were tested, such as MMP-9, IL-1 $\beta$, CXCL1/KC, CXCL2/MIP-2, and IL-6. MMP-9 is thought to be a major effector of tissue destruction caused by vesicants and may contribute to blister formation by separating the dermis from the epidermis $(49,50) . \mathrm{KC}$ is a potent neutrophil attractant, guiding pro-inflammatory neutrophils into the injured tissue. IL-1 $\beta$ is produced by macrophages and various other cell types in response to inflammatory agents, infections, or microbial endotoxins. IL-1 $\beta$ is not produced in healthy tissues, with the exception of skin keratinocytes, some epithelial cells, and certain cells of the nervous system. In our studies, we observed similar findings that support the expression of IL-1 $\beta$ in control ears. In these studies, although inflammatory cytokine makers were decreased in treatment groups compared to the vehicle group, some variability was observed between SPMs tested.

We developed an in vivo imaging with infra-red fluorescent dye to visualize extravasation of inflammatory exudate and real-time healing. Although such imaging is done frequently in tumor biology studies, this is the first of its kind in vivo imaging technique in cutaneous inflammatory studies (51). Increased vascular leakage was noted in mouse ears exposed to CEES whereas treatment with SPMs decreased vascular leakage. Vascular leakage was quantified with the intensity of fluorescence. Treatment with SPMs significantly decreased fluorescence of IRDye 800 CW PEG. Vascular leakage is a common feature of inflammation. Vascular permeability is a result of increased capillary hydrostatic pressure and increased spaces between vascular endothelial linings. The non-specific fluorescent dye, IRDye 800CW PEG contrast agent $(25-60 \mathrm{kDa})$, used in this study reaches the sites of acute inflammation due to vascular leakage and decreased return to the vasculature due to alteration in colloid osmotic pressure. We visualized ear vasculature in mice as early as 10 minutes post-administration of dye. The method was optimized to track the progression of inflammation in real time with this less invasive procedure. In this study, we injected IRDye 800CW PEG agent at 1:2 dilution (0.5 nmol per animal) instead of the manufacturer's suggested amount (1 nmol per animal) to avoid signal saturation. IRDye $800 \mathrm{CW}$ is excreted in urine. Therefore, temporary individual caging of mice with cage enrichment after the injection of dye is suggested to prevent background noise from urine excreted by the cage mates. This technique is highly useful in tracking the therapeutic efficacy of potential drug candidates in long-term skin injury studies.

\section{CS tear gas}

In recent years, the deployment of CS tear gas agent has significantly increased worldwide, including the United States for riot control. Although tear gas agents have been used since several decades for riot control and to incapacitate enemies in war fronts, no specific therapeutic agents are 
available for treating injuries caused by tear gas agents. In a real riot control situation, the actual concentration of exposure of CS tear agent is unknown and is highly variable. In our in vivo CS tear gas agent exposure titration studies, we found that exposure to concentrations of 50 - $200 \mathrm{mM}$ of CS tear gas agent saturated the response. However, in this study, we tested the therapeutic potential of pro-resolving agents at $200 \mathrm{mM}$ concentration $(20 \mu \mathrm{L})$ of CS tear agent to show therapeutic benefits even at higher CS tear gas exposure levels.

The clinical manifestation of CS tear gas exposure includes immediate burning and pain sensation. These signs are the result of intense sensory irritation at various body sites. Studies in our laboratory have shown that CS tear gas exerts its effect through the activation of TRPA1 channels $(8$, 52). After the removal of patients from CS tear gas exposure site, lacrimation and burning sensation may decrease within 30 minutes. However, exposure to higher concentrations of this xenobiotic or for a prolonged period will result in severe cutaneous injuries.

In this study, edema and erythema were the most significant findings of cutaneous injury upon exposure to CS tear gas agent. Both ear punch biopsy gross markers and histopathology demonstrated heightened edema with CS tear gas exposure. Treatment with SPMs significantly reduced inflammation. Similar anti-edematogenic effects of SPMs were observed in carrageenan-induced paw edema (53). Proinflammatory markers were significantly increased in CS tear gas agent exposed mice whereas treatment with pro-resolving agents decreased these cytokine markers.

\section{Dose and route of administration}

The results from this study were promising in showing the therapeutic effects of SPMs in two chemical threat agents-induced cutaneous inflammation. There are several teams studying the potential use of these agents as therapeutics against several indications around the world $(30,32,33,36,38,39$, 47, 54-57) . Based on previous studies in other disease modalities in mouse models, nano-gram amounts were administered in these studies and these doses were shown to be therapeutically effective. In this study, therapeutic efficacies of pro-resolving agents were demonstrated in the intraperitoneal route of administration. However, in mass casualty situations, auto-injectors for intramuscular injection or oral medication would be ideal. Testing the efficacy of pro-resolving agents through other routes of administration, such as intramuscular and subcutaneous are warranted. It is expected that the intramuscular and subcutaneous route of administration would be more efficacious compared to the variable intraperitoneal route of administration. 
In the continuum of these studies, testing therapeutic potential in other routes of administration, testing combination of pro-resolving agents to get the synergistic beneficial effects and testing in higher mammalian species are warranted. Additional pro-resolving agents are discovered recently and it's worth to screen therapeutic potential of these new agents in chemical cutaneous injury models.

\section{Mechanism of action of SPM}

Although the mechanism of action of pro-resolving agents in this study was not elucidated, the published literature shows several possible mechanisms of action $(30,55,58)$. Recent studies show that pro-resolving agents work through $\mathrm{G}$ protein-coupled receptors (GPCRs), such as ALX/FPR2, GPR18 and GPR32, whereas a few other studies show that these agents work through inhibition of transient receptor potential channels $(35,42,59-61)$. Achanta et al., Bessac et al., and Stenger et al., have shown the involvement of transient receptor potential ankyrin repeat 1 (TRPA1) ion channel in mediating the effects of CEES and CS tear gas agents using in vitro and in vivo studies (6, 52, 62-64). Park et al and others showed that RvD1 and RvD2 regulate TRPA1 currents in mouse DRG neurons $(35,59,65)$. Based on the literature review, we believe that SPMs mitigated the cutaneous injury caused by CEES and CS tear gas agents through inhibition of TRPA1 ion channels (Figure 7). The interactions between GPCRs and TRP ion channels further substantiate our proposed mechanism of action of SPMs (RvD1 or RvD2) through inhibition of TRPA1 ion channels $(66,67)$. However, further in vitro studies with TRPA1 transfection in HEK293T cells or in vivo studies with TRPA1 knock mouse are warranted to test our interpretation.

\section{Potential of pro-resolving agents as therapeutic candidates:}

Due to the similarity of SPMs to endogenous lipid mediators, and because of the low dosages required to initiate resolution of inflammation, it is expected that these agents will display minimal side effects or toxicity compared to the conventional NSAIDs or opioids. No side effects or toxicity of SPMs was published in the literature. A combination of these SPMs might also offer better therapeutic outcomes compared to single-agent therapy which is worth testing. Few of the limitations of SPMs are lack of stability at room temperature and non-availability of commercial SPMs as drug formulations. Some of the commercial nutraceuticals enriched with metabolites of EPA and DHA such as 17-hydroxydocosahexaenoic acid (17-HDHA), 18-hydroxy-eicosapentaenoic acid (18-HEPE), and 14-DDHA might be worthwhile to test in the skin injury models for their efficacy (https://www.metagenics.com/spmactive, accessed 02/11/2019; http://www.solutex.es/en/products/biolipidos/lipinova, accessed 
02/11/2019). If the therapeutic potential of these SPMs is established in rodent and non-rodent higher mammalian species, then the approval will be feasible for the indications of sulfur mustard and tear gas agent-induced cutaneous inflammation under the US FDA's animal rule, unlike other new chemical entities (https://www.fda.gov/downloads/drugs/guidances/ucm399217.pdf, accessed 10/23/2018).

\section{Conclusion}

Exposure of CS tear gas agent and CEES to ear skin caused profound cutaneous inflammation in mouse models. Post-exposure treatment with specialized pro-resolving mediators decreased inflammation. These SPMs (RvD1 and RvD2) can be used as potential medical countermeasures to hasten the resolution of inflammation caused by chemical threat agents. 


\section{Acknowledgments}

We appreciate thoughtful discussions of Drs. Bruce Levy, MD and Charles Serhan, PhD, Harvard University, Boston, MA, USA. This study was supported by grants from NIH (NIEHS): R21ES02287501 (awarded to SEJ). The content is solely the responsibility of the author and does not necessarily represent the views of the NIH.

\section{Conflict of interest}

Authors do not have any conflicts of interests to disclose

\section{Author contributions}

SA, NC, and SEJ designed research; SA, NC, BL, and SB performed research; SA and NC conducted in vivo studies independently. SA developed and optimized new in vivo imaging with an infrared fluorescent dye; SA, NC, and SEJ analyzed data; SA wrote the first draft of manuscript; SA and SEJ approved the final version. 


\section{Figure legends:}

Figure 1. Gross morphological parameters and histopathology in CEES-exposed mice, treated with Resolvin D1 (RvD1) or vehicle.

A) 2-chloroethyl-ethyl-sulfide mouse ear vesicant model (CEES MEVM) and treatment regimen. Right and left ears of mice were exposed to CEES and vehicle (dichloromethane), respectively. RvD1 (2 $\mu \mathrm{g} / \mathrm{kg}$ i.p) or vehicle was administered at $1 \mathrm{~h}$ post-CEES exposure.

B) Ear thickness and ear punch biopsy weights. Ear thickness was measured by spring-loaded calipers. Ear punch biopsy weights were measured from three $4 \mathrm{~mm}$ circular ear punch biopsy samples. Percent decrease or increase in ear thickness and ear punch biopsy weights over control is presented.

C) Representative H\&E stained ear punch biopsy histopathology profiles are presented.

Data are presented as mean \pm SEM. $n=5$ /group.

Figure 2. Therapeutic effects of Resolvin D1 on vascular leakage and pro-inflammatory cytokines in ear punch biopsy homogenates of CEES-exposed mice. 2-chloroethyl-ethyl-sulfide mouse ear vesicant model (CEES MEVM) and treatment regimen are as in Figure 1A.

A) Representative images of vascular leakage in CEES MEVM. Extravasation of inflammatory exudate into surrounding tissues and the resolution of inflammation with the administration of RvD1 or vehicle in CEES-exposed mice was studied by injecting IRDye $800 \mathrm{CW}$ intravenously at $18 \mathrm{~h}$ post-CEES exposure. Mice were scanned at $23 \mathrm{~h}$ post-CEES exposure using Li-Cor Odyssey CLX ${ }^{\circledR}$.

B) The percent increase or decrease in intensity of dye in the CEES-exposed ear over control ear is presented ( $\mathrm{n}=5 /$ group).

C) Expression of MMP-9 ( $\mathrm{n}=10-11 /$ group) and pro-inflammatory cytokines such as IL-1 $\beta$ (5-9/group) and CXCL1/KC (4-8/group) in ear punch biopsy homogenate samples using ELISA.

Data are presented as mean \pm SEM.

Figure 3. Gross morphological parameters and histopathology in CEES-exposed mice, treated with Resolvin D2 (RvD2) or vehicle.

2-chloroethyl-ethyl-sulfide mouse ear vesicant model (CEES MEVM) and treatment regimen are as in Figure 1A. Mice received either Resolvin D2 $(\operatorname{RvD} 2)$ at a dose rate of $2 \mu \mathrm{g} / \mathrm{kg}$ i.p body weight or 
vehicle $1 \mathrm{~h}$ post-CEES exposure. A) Ear thickness and ear punch biopsy weights. Ear thickness was measured using spring-loaded calipers. Ear punch biopsy weights were determined from three $4 \mathrm{~mm}$ ear punch biopsy samples from each ear. Percent increase or decrease in ear thickness and ear punch biopsy weights over control are presented. B) Representative H\&E stained ear punch biopsy histopathology samples are presented.

Data are presented as mean \pm SEM. $n=10 /$ group.

Figure 4. Therapeutic effects of Resolvin D2 on vascular leakage and pro-inflammatory cytokines in ear punch biopsy homogenates of CEES-exposed mice. 2-chloroethyl-ethyl-sulfide mouse ear vesicant model (CEES MEVM) and treatment regimen are as in Figure 1A.

A) Representative images of vascular leakage in CEES MEVM. Extravasation of inflammatory exudate into surrounding tissues and the resolution of inflammation with the administration of RvD2 or vehicle in CEES-exposed mice was studied by injecting IRDye 800CW intravenously at $18 \mathrm{~h}$ post-CEES exposure. Mice were scanned at 23 h post-CEES exposure using Li-Cor Odyssey CLX ${ }^{\circledR}$. B) The percent increase or decrease in the intensity of dye in the CEES-exposed over control ears is presented $(n=3$ 4/group). C) Expression of pro-inflammatory cytokines such as CXCL2/MIP-2, IL-1 $\beta$ and CXCL1/KC in ear punch biopsy homogenate samples using ELISA ( $n=3-6 /$ group). Data as mean \pm SEM.

Figure 5. Therapeutic effects of Resolvin D1 (RvD1) in CS tear gas-induced acute skin inflammation. A) CS tear gas exposure and treatment regimen. Right and left ears of mice were exposed to CS tear gas agent and vehicle (DMSO), respectively. Mice received either RvD1 at a dose rate of $5 \mu \mathrm{g} / \mathrm{kg}$ body weight at 30 minutes and 4-hour post-CS tear gas exposure or vehicle $(0.1 \%$ ethanol in PBS) intraperitoneally. B) Ear thickness and ear punch biopsy weights. Ear thickness was measured by spring-loaded calipers. Ear punch biopsy weights were measured from three 4 mm circular ear punch biopsy samples. Percent decrease or increase in ear thickness and ear punch biopsy weights over control is presented (n=9-10/group). C) Representative H\&E stained ear punch biopsy histopathology samples are presented. D) Expression of MMP-9 and pro-inflammatory cytokines such as IL-1 $\beta$ and CXCL1/KC in ear punch biopsy homogenate samples using ELISA (n=3-8/group).

Data are presented as mean \pm SEM.

Figure 6. Therapeutic effects of Resolvin D2 (RvD2) in CS tear gas-induced acute skin inflammation. CS tear gas exposure and treatment regimen are as in Figure 5A. Mice received either 
RvD2 at a dose rate of $5 \mu \mathrm{g} / \mathrm{kg}$ body weight at 30 minutes and 4-hour post-CS tear gas exposure or vehicle $(0.1 \%$ ethanol in PBS) intraperitoneally.

A) Ear thickness and ear punch biopsy weights. Ear thickness was measured by spring-loaded calipers. Ear punch biopsy weights were measured from three $4 \mathrm{~mm}$ circular ear punch biopsy samples. Percent decrease or increase in ear thickness and ear punch biopsy weights over control is presented ( $n=10 /$ group). B) Representative H\&E stained ear punch biopsy histopathology samples are presented. C) Expression of pro-inflammatory cytokines such as IL-6, CXCL1/KC, and MIP-2 in ear punch biopsy homogenate samples using ELISA ( $n=4-6 /$ group). Data are presented as mean \pm SEM.

Figure 7. Mechanism of action of SPMs through GPCR-TRP ion channel axis. Transient receptor potential Ankyrin repeat 1 (TRPA1) ion channels mediate the effects of CEES and CS tear gas agents. SPMs (RvD1 or RvD2) ameliorate the inflammation caused by CEES and CS tear agents through inhibition of TRPA1 ion channels directly or through modulation of G-protein coupled receptors (GPCRs) which subsequently inhibits TRPA1 ion channels. 


\section{References}

1. Etezad-Razavi, M., Mahmoudi, M., Hefazi, M., and Balali-Mood, M. (2006) Delayed ocular complications of mustard gas poisoning and the relationship with respiratory and cutaneous complications. Clinical \& experimental ophthalmology 34, 342-346

2. Ghanei, M., Poursaleh, Z., Harandi, A. A., Emadi, S. E., and Emadi, S. N. (2010) Acute and chronic effects of sulfur mustard on the skin: a comprehensive review. Cutaneous and ocular toxicology 29, 269-277

3. McGovern, T., Day, B. J., White, C. W., Powell, W. S., and Martin, J. G. (2011) AEOL10150: a novel therapeutic for rescue treatment after toxic gas lung injury. Free Radic Biol Med 50, 602-608

4. Sunil, V. R., Patel-Vayas, K., Shen, J., Gow, A. J., Laskin, J. D., and Laskin, D. L. (2011) Role of TNFR1 in lung injury and altered lung function induced by the model sulfur mustard vesicant, 2-chloroethyl ethyl sulfide. Toxicol Appl Pharmacol 250, 245-255

5. Jain, A. K., Tewari-Singh, N., Gu, M., Inturi, S., White, C. W., and Agarwal, R. (2011) Sulfur mustard analog, 2-chloroethyl ethyl sulfide-induced skin injury involves DNA damage and induction of inflammatory mediators, in part via oxidative stress, in SKH-1 hairless mouse skin. Toxicol Lett 205, 293301

6. Achanta, S., Chintagari, N. R., Brackmann, M., Balakrishna, S., and Jordt, S. E. (2018) TRPA1 and CGRP antagonists counteract vesicant-induced skin injury and inflammation. Toxicol Lett

7. Balali-Mood, M., Riahi-Zanjani, B., Mahmoudi, M., and Sadeghi, M. (2019) Current status of the acquired immune system of Iranian patients with long-term complications of sulfur mustard poisoning. Daru

8. Rothenberg, C., Achanta, S., Svendsen, E. R., and Jordt, S. E. (2016) Tear gas: an epidemiological and mechanistic reassessment. Ann $N$ Y Acad Sci

9. Schep, L. J., Slaughter, R. J., and McBride, D. I. (2013) Riot control agents: the tear gases CN, CS and OC-a medical review. J R Army Med Corps

10. Southward, R. D. (2001) Cutaneous burns from CS incapacitant spray. Medicine, science, and the law 41, 74-77

11. Schlund, S., Mladenovic, M., Basilio Janke, E. M., Engels, B., and Weisz, K. (2005) Geometry and cooperativity effects in adenosine-carboxylic acid complexes. J Am Chem Soc 127, 16151-16158

12. Bhargava, K., Banerjee, P., and White, I. R. (2012) Investigating contact allergy to CS spray. Contact dermatitis 66, 109-110

13. Shambhu, S., and Kurtis, R. (2011) Allergic contact dermatitis due to CS spray. Emergency medicine journal : EMJ 28, 345

14. Kain, N., Mishra, A., and James, M. I. (2010) Guidance needed on secondary effects of CS gas on staff. $B M J$ 340, C1189

15. Karagama, Y. G., Newton, J. R., and Newbegin, C. J. (2003) Short-term and long-term physical effects of exposure to CS spray. Journal of the Royal Society of Medicine 96, 172-174

16. Karaman, E., Erturan, S., Duman, C., Yaman, M., and Duman, G. U. (2009) Acute laryngeal and bronchial obstruction after CS (o-chlorobenzylidenemalononitrile) gas inhalation. European archives of oto-rhinolaryngology : official journal of the European Federation of Oto-Rhino-Laryngological Societies 266, 301304

17. McGovern, T. K., Goldberger, M., Allard, B., Farahnak, S., Hamamoto, Y., O'Sullivan, M., Hirota, N., Martel, G., Rousseau, S., and Martin, J. G. (2015) Neutrophils mediate airway hyperresponsiveness after chlorine-induced airway injury in the mouse. Am J Respir Cell Mol Biol 52, 513-522

18. Rose, D., Schmidt, A., Brandenburger, M., Sturmheit, T., Zille, M., and Boltze, J. (2018) Sulfur mustard skin lesions: A systematic review on pathomechanisms, treatment options and future research directions. Toxicol Lett 293, 82-90

19. Poursaleh, Z., Ghanei, M., Babamahmoodi, F., Izadi, M., Harandi, A. A., Emadi, S. E., Taghavi, N. O., Sayad-Nouri, S. S., and Emadi, S. N. (2012) Pathogenesis and treatment of skin lesions caused by sulfur mustard. Cutaneous and ocular toxicology 31, 241-249

20. Carron, P. N., and Yersin, B. (2009) Management of the effects of exposure to tear gas. BMJ 338, b2283 
21. Luka, A., Stolbach, A., and Hoffman, R. S. (2007) Response to "Prevention of CS 'tear gas' eye and skin effects and active decontamination with diphoterine: preliminary studies in 5 French Gendarmes". The Journal of emergency medicine 32, 309-310; author reply 310-301

22. Viala, B., Blomet, J., Mathieu, L., and Hall, A. H. (2005) Prevention of CS "tear gas" eye and skin effects and active decontamination with Diphoterine: preliminary studies in 5 French Gendarmes. The Journal of emergency medicine 29, 5-8

23. Jones, G. R. (1996) CS sprays: antidote and decontaminant. Lancet 347, 968-969

24. Kadar, T., Fishbeine, E., Meshulam, Y., Sahar, R., Chapman, S., Liani, H., Barness, I., and Amir, A. (2000) Treatment of skin injuries induced by sulfur mustard with calmodulin antagonists, using the pig model. $J$ Appl Toxicol 20 Suppl 1, S133-136

25. Laskin, J. D., Black, A. T., Jan, Y. H., Sinko, P. J., Heindel, N. D., Sunil, V., Heck, D. E., and Laskin, D. L. (2010) Oxidants and antioxidants in sulfur mustard-induced injury. Ann N Y Acad Sci 1203, 92-100

26. Paromov, V., Suntres, Z., Smith, M., and Stone, W. L. (2007) Sulfur mustard toxicity following dermal exposure: role of oxidative stress, and antioxidant therapy. Journal of burns and wounds 7, e7

27. Tewari-Singh, N., Inturi, S., Jain, A. K., Agarwal, C., Orlicky, D. J., White, C. W., Agarwal, R., and Day, B. J. (2014) Catalytic antioxidant AEOL 10150 treatment ameliorates sulfur mustard analog 2-chloroethyl ethyl sulfide-associated cutaneous toxic effects. Free Radic Biol Med 72, 285-295

28. Tewari-Singh, N., Jain, A. K., Inturi, S., Agarwal, C., White, C. W., and Agarwal, R. (2012) Silibinin attenuates sulfur mustard analog-induced skin injury by targeting multiple pathways connecting oxidative stress and inflammation. PloS one 7, e46149

29. Haworth, O., and Levy, B. D. (2007) Endogenous lipid mediators in the resolution of airway inflammation. The European respiratory journal 30, 980-992

30. Buckley, C. D., Gilroy, D. W., and Serhan, C. N. (2014) Proresolving lipid mediators and mechanisms in the resolution of acute inflammation. Immunity 40, 315-327

31. Dalli, J. (2017) Does promoting resolution instead of inhibiting inflammation represent the new paradigm in treating infections? Mol Aspects Med 58, 12-20

32. Serhan, C. N. (2017) Treating inflammation and infection in the 21st century: new hints from decoding resolution mediators and mechanisms. FASEB J 31, 1273-1288

33. Serhan, C. N., and Levy, B. D. (2018) Resolvins in inflammation: emergence of the pro-resolving superfamily of mediators. $J$ Clin Invest

34. Ji, R. R., Xu, Z. Z., Strichartz, G., and Serhan, C. N. (2011) Emerging roles of resolvins in the resolution of inflammation and pain. Trends Neurosci 34, 599-609

35. Park, C. K., Xu, Z. Z., Liu, T., Lu, N., Serhan, C. N., and Ji, R. R. (2011) Resolvin D2 is a potent endogenous inhibitor for transient receptor potential subtype V1/A1, inflammatory pain, and spinal cord synaptic plasticity in mice: distinct roles of resolvin D1, D2, and E1. The Journal of neuroscience : the official journal of the Society for Neuroscience 31, 18433-18438

36. Levy, B. D., Hickey, L., Morris, A. J., Larvie, M., Keledjian, R., Petasis, N. A., Bannenberg, G., and Serhan, C. N. (2005) Novel polyisoprenyl phosphates block phospholipase $D$ and human neutrophil activation in vitro and murine peritoneal inflammation in vivo. Br J Pharmacol 146, 344-351

37. Maderna, P., and Godson, C. (2009) Lipoxins: resolutionary road. Br J Pharmacol 158, 947-959

38. Aoki, H., Hisada, T., Ishizuka, T., Utsugi, M., Kawata, T., Shimizu, Y., Okajima, F., Dobashi, K., and Mori, M. (2008) Resolvin E1 dampens airway inflammation and hyperresponsiveness in a murine model of asthma. Biochem Biophys Res Commun 367, 509-515

39. Aoki, H., Hisada, T., Ishizuka, T., Utsugi, M., Ono, A., Koga, Y., Sunaga, N., Nakakura, T., Okajima, F., Dobashi, K., and Mori, M. (2010) Protective effect of resolvin E1 on the development of asthmatic airway inflammation. Biochem Biophys Res Commun 400, 128-133

40. Seki, H., Fukunaga, K., Arita, M., Arai, H., Nakanishi, H., Taguchi, R., Miyasho, T., Takamiya, R., Asano, K., Ishizaka, A., Takeda, J., and Levy, B. D. (2010) The anti-inflammatory and proresolving mediator resolvin E1 protects mice from bacterial pneumonia and acute lung injury. $J$ Immunol 184, 836-843

41. Ballard-Croft, C., Wang, D., Sumpter, L. R., Zhou, X., and Zwischenberger, J. B. (2012) Large-animal models of acute respiratory distress syndrome. Ann Thorac Surg 93, 1331-1339 
42. Bang, S., Yoo, S., Yang, T. J., Cho, H., and Hwang, S. W. (2012) 17(R)-resolvin D1 specifically inhibits transient receptor potential ion channel vanilloid 3 leading to peripheral antinociception. Br J Pharmacol 165, 683-692

43. Bento, A. F., Claudino, R. F., Dutra, R. C., Marcon, R., and Calixto, J. B. (2011) Omega-3 fatty acid-derived mediators 17(R)-hydroxy docosahexaenoic acid, aspirin-triggered resolvin D1 and resolvin D2 prevent experimental colitis in mice. J Immunol 187, 1957-1969

44. Lima-Garcia, J. F., Dutra, R. C., da Silva, K., Motta, E. M., Campos, M. M., and Calixto, J. B. (2011) The precursor of resolvin $D$ series and aspirin-triggered resolvin D1 display anti-hyperalgesic properties in adjuvant-induced arthritis in rats. Br J Pharmacol 164, 278-293

45. Norling, L. V., Spite, M., Yang, R., Flower, R. J., Perretti, M., and Serhan, C. N. (2011) Cutting edge: Humanized nano-proresolving medicines mimic inflammation-resolution and enhance wound healing. $J$ Immunol 186, 5543-5547

46. Xu, J., Duan, X., Hu, F., Poorun, D., Liu, X., Wang, X., Zhang, S., Gan, L., He, M., Zhu, K., Ming, Z., and Chen, H. (2018) Resolvin D1 attenuates imiquimod-induced mice psoriasiform dermatitis through MAPKs and NF-kappaB pathways. Journal of dermatological science 89, 127-135

47. Zhang, L. Y., Jia, M. R., and Sun, T. (2018) The roles of special proresolving mediators in pain relief. Rev Neurosci 29, 645-660

48. Silny, W., Czarnecka-Operacz, M., and Silny, P. (2005) The new scoring system for evaluation of skin inflammation extent and severity in patients with atopic dermatitis. Acta Dermatovenerol Croat 13, 219224

49. Malaviya, R., Sunil, V. R., Cervelli, J., Anderson, D. R., Holmes, W. W., Conti, M. L., Gordon, R. E., Laskin, J. D., and Laskin, D. L. (2010) Inflammatory effects of inhaled sulfur mustard in rat lung. Toxicol Appl Pharmacol 248, 89-99

50. Shakarjian, M. P., Bhatt, P., Gordon, M. K., Chang, Y. C., Casbohm, S. L., Rudge, T. L., Kiser, R. C., Sabourin, C. L., Casillas, R. P., Ohman-Strickland, P., Riley, D. J., and Gerecke, D. R. (2006) Preferential expression of matrix metalloproteinase- 9 in mouse skin after sulfur mustard exposure. J Appl Toxicol 26, 239-246

51. Vasey, P. A., Kaye, S. B., Morrison, R., Twelves, C., Wilson, P., Duncan, R., Thomson, A. H., Murray, L. S., Hilditch, T. E., Murray, T., Burtles, S., Fraier, D., Frigerio, E., and Cassidy, J. (1999) Phase I clinical and pharmacokinetic study of PK1 [N-(2-hydroxypropyl)methacrylamide copolymer doxorubicin]: first member of a new class of chemotherapeutic agents-drug-polymer conjugates. Cancer Research Campaign Phase I/II Committee. Clinical cancer research : an official journal of the American Association for Cancer Research 5, 83-94

52. Bessac, B. F., Sivula, M., von Hehn, C. A., Caceres, A. I., Escalera, J., and Jordt, S. E. (2009) Transient receptor potential ankyrin 1 antagonists block the noxious effects of toxic industrial isocyanates and tear gases. FASEB J 23, 1102-1114

53. Menezes-de-Lima, O., Jr., Kassuya, C. A., Nascimento, A. F., Henriques, M., and Calixto, J. B. (2006) Lipoxin A4 inhibits acute edema in mice: implications for the anti-edematogenic mechanism induced by aspirin. Prostaglandins Other Lipid Mediat 80, 123-135

54. de Paiva, C. S., Schwartz, C. E., Gjorstrup, P., and Pflugfelder, S. C. (2012) Resolvin E1 (RX-10001) reduces corneal epithelial barrier disruption and protects against goblet cell loss in a murine model of dry eye. Cornea 31, 1299-1303

55. Ishizuka, T., Hisada, T., Aoki, H., and Mori, M. (2008) Resolvin E1: a novel lipid mediator in the resolution of allergic airway inflammation. Expert review of clinical immunology 4, 669-672

56. Zhang, L., Terrando, N., Xu, Z. Z., Bang, S., Jordt, S. E., Maixner, W., Serhan, C. N., and Ji, R. R. (2018) Distinct Analgesic Actions of DHA and DHA-Derived Specialized Pro-Resolving Mediators on Postoperative Pain After Bone Fracture in Mice. Front Pharmacol 9, 412

57. Zheng, S., Wang, Q., D'Souza, V., Bartis, D., Dancer, R., Parekh, D., Gao, F., Lian, Q., Jin, S., and Thickett, D. R. (2018) ResolvinD1 stimulates epithelial wound repair and inhibits TGF-beta-induced EMT whilst reducing fibroproliferation and collagen production. Lab Invest 98, 130-140 
58. Haworth, O., Cernadas, M., and Levy, B. D. (2011) NK cells are effectors for resolvin E1 in the timely resolution of allergic airway inflammation. J Immunol 186, 6129-6135

59. Bang, S., Yoo, S., Oh, U., and Hwang, S. W. (2010) Endogenous lipid-derived ligands for sensory TRP ion channels and their pain modulation. Arch Pharm Res 33, 1509-1520

60. Norling, L. V., and Serhan, C. N. (2010) Profiling in resolving inflammatory exudates identifies novel antiinflammatory and pro-resolving mediators and signals for termination. Journal of internal medicine 268, 15-24

61. Agarwal, S., Sane, R., Ohlfest, J. R., and Elmquist, W. F. (2011) The role of the breast cancer resistance protein (ABCG2) in the distribution of sorafenib to the brain. J Pharmacol Exp Ther 336, 223-233

62. Stenger, B., Popp, T., John, H., Siegert, M., Tsoutsoulopoulos, A., Schmidt, A., Muckter, H., Gudermann, T., Thiermann, H., and Steinritz, D. (2017) N-Acetyl-L-cysteine inhibits sulfur mustard-induced and TRPA1-dependent calcium influx. Arch Toxicol 91, 2179-2189

63. Stenger, B., Zehfuss, F., Muckter, H., Schmidt, A., Balszuweit, F., Schafer, E., Buch, T., Gudermann, T., Thiermann, H., and Steinritz, D. (2015) Activation of the chemosensing transient receptor potential channel A1 (TRPA1) by alkylating agents. Arch Toxicol 89, 1631-1643

64. Achanta, S., and Jordt, S. E. (2017) TRPA1: Acrolein meets its target. Toxicol Appl Pharmacol 324, 45-50

65. Bang, S., Yoo, S., Yang, T. J., Cho, H., Kim, Y. G., and Hwang, S. W. (2010) Resolvin D1 attenuates activation of sensory transient receptor potential channels leading to multiple anti-nociception. $\mathrm{Br} J$ Pharmacol 161, 707-720

66. Veldhuis, N. A., Poole, D. P., Grace, M., McIntyre, P., and Bunnett, N. W. (2015) The G protein-coupled receptor-transient receptor potential channel axis: molecular insights for targeting disorders of sensation and inflammation. Pharmacol Rev 67, 36-73

67. Yekkirala, A. S. (2013) Two to tango: GPCR oligomers and GPCR-TRP channel interactions in nociception. Life Sci 92, 438-445 


\section{Figure 2}

A
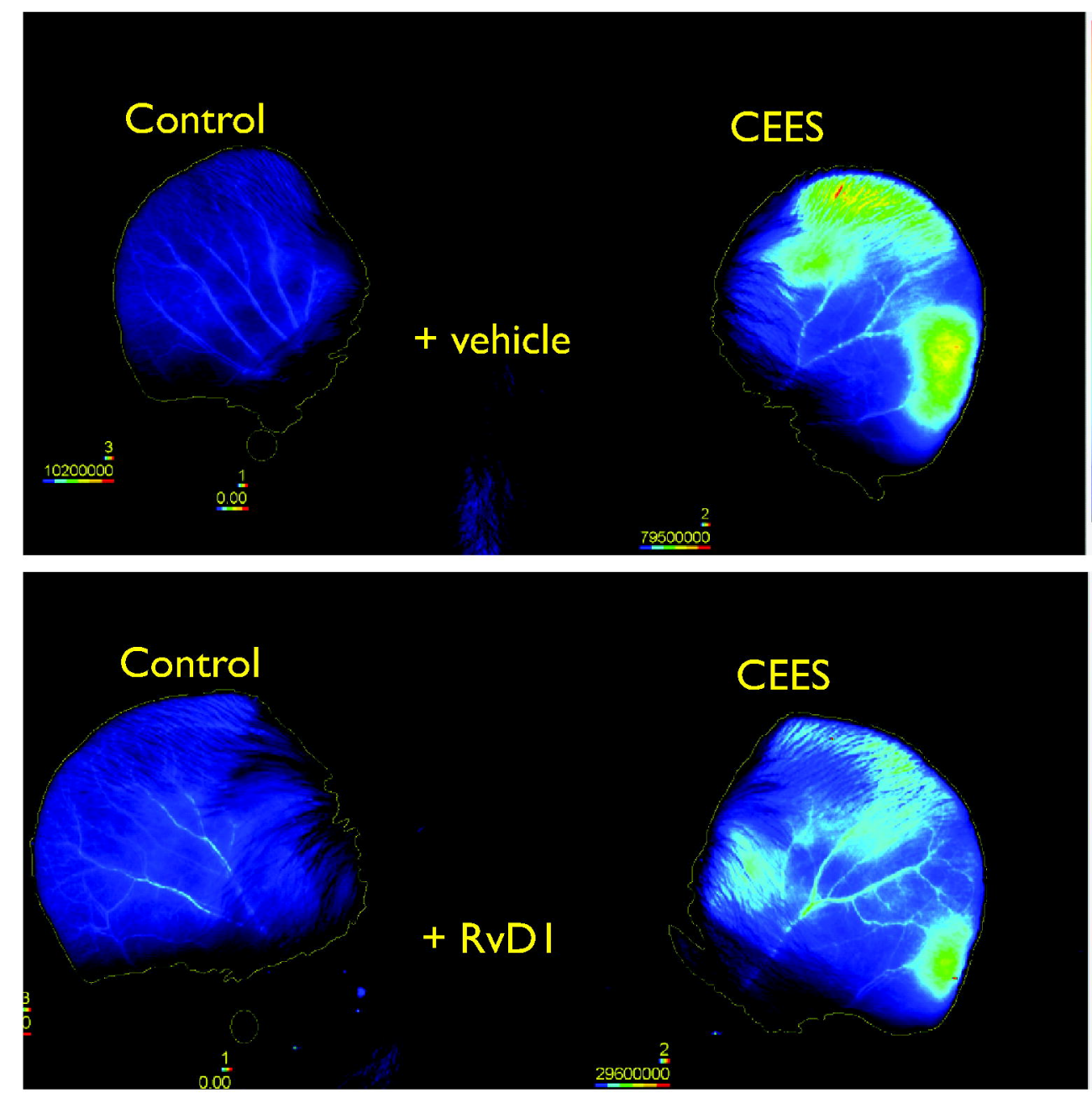

Control

$+\operatorname{RvDl}$
B

\section{IRDye 800CW PEG}

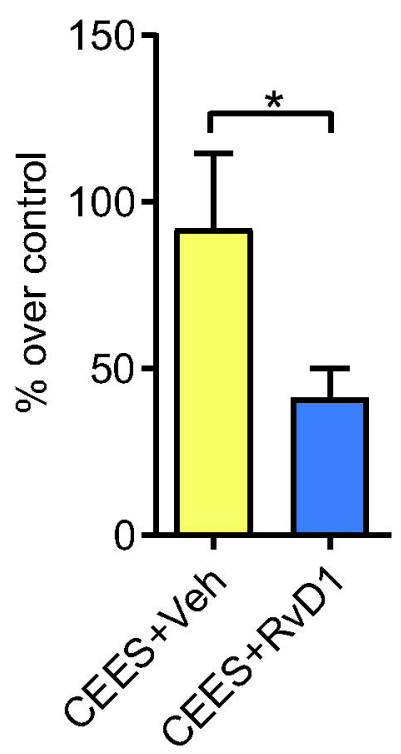

C

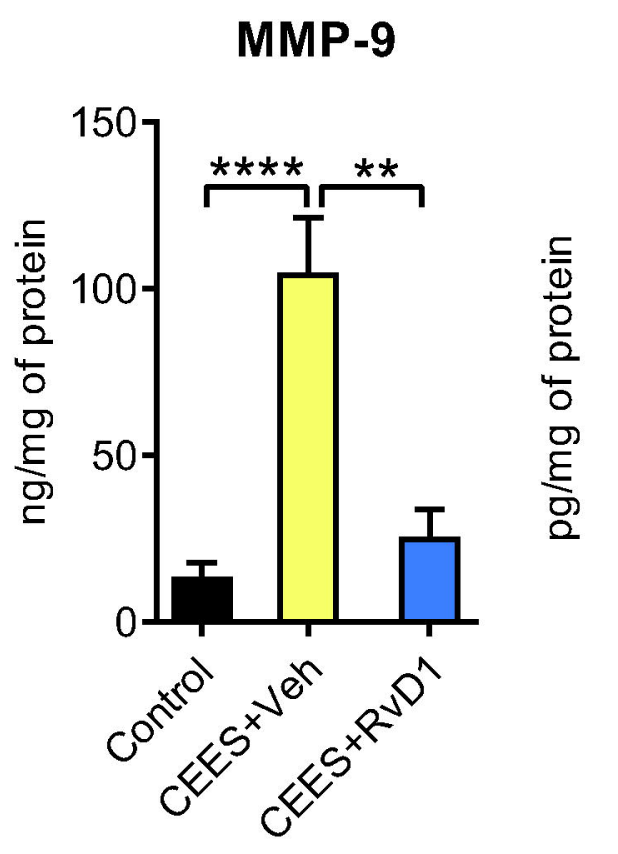

\section{IL1-beta}
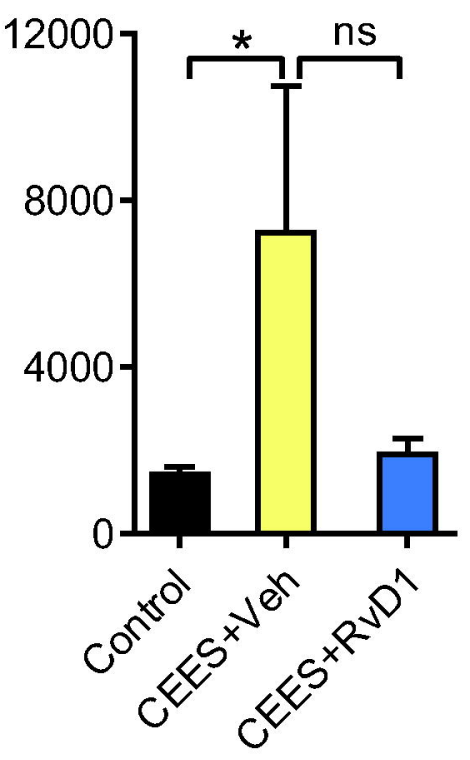

CXCL1/KC

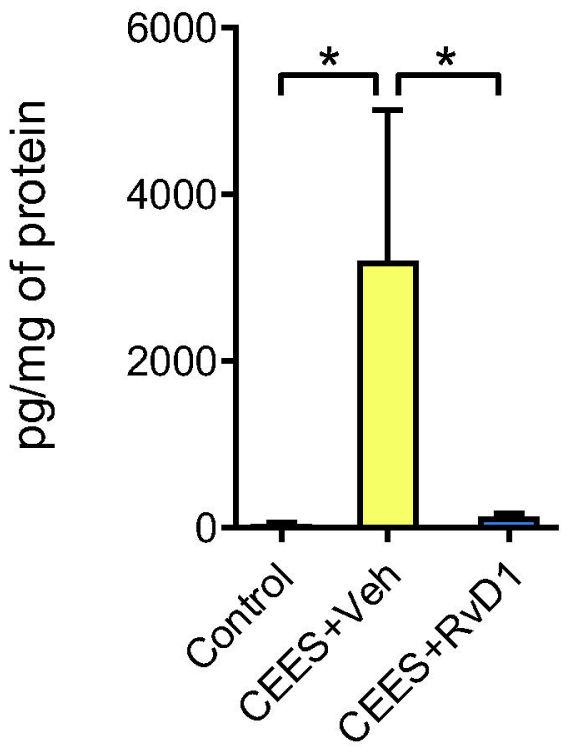


Figure 3

\section{A Ear thickness}

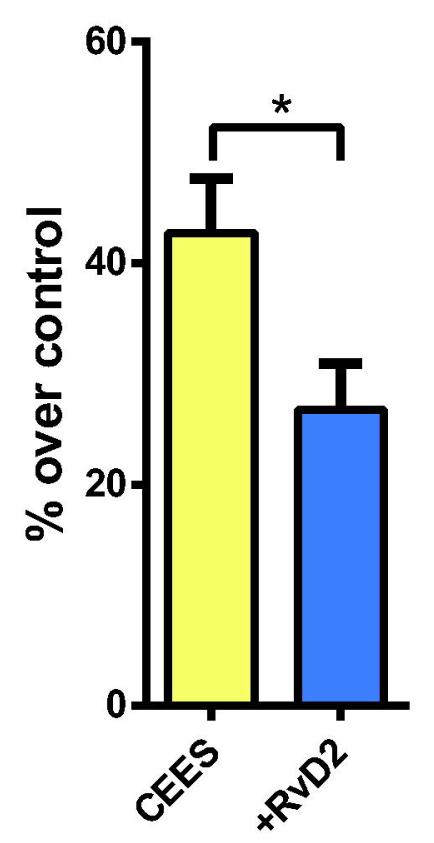

\section{Ear punch biopsy weights}

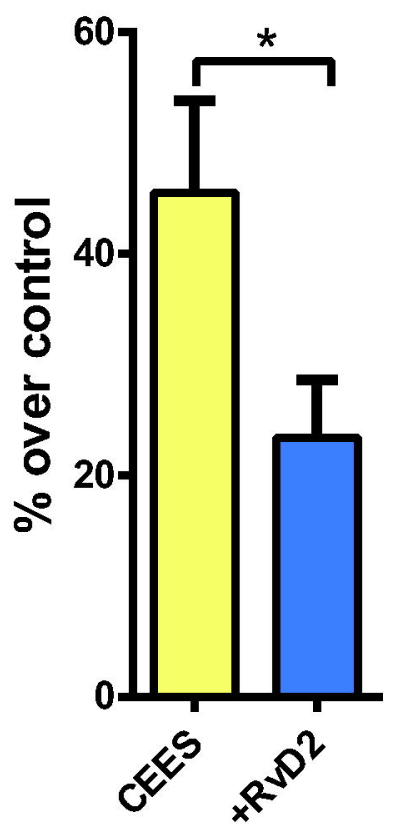

B

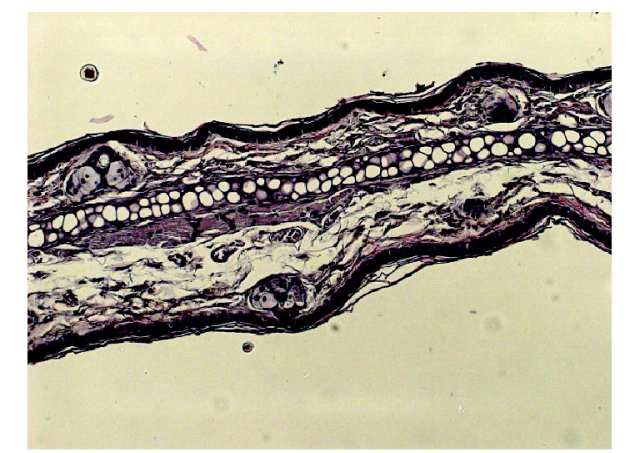

Control

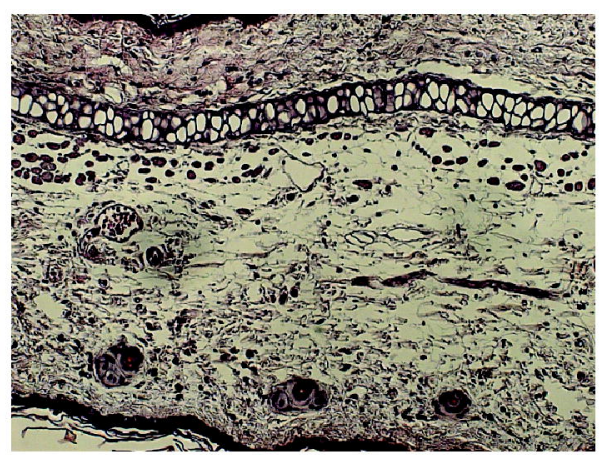

CEES

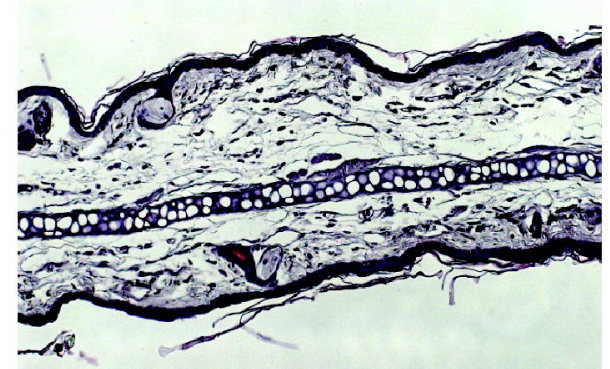

CEES+RvD2 


\section{Figure 4}

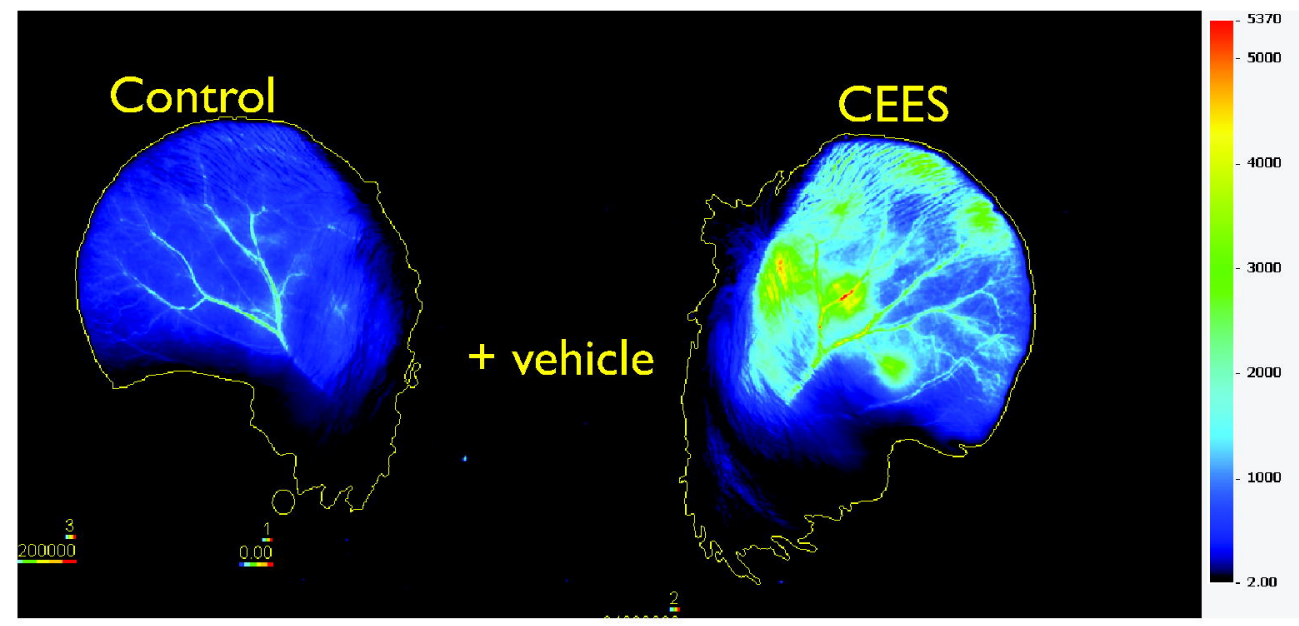

\section{IRDye 800CW PEG}

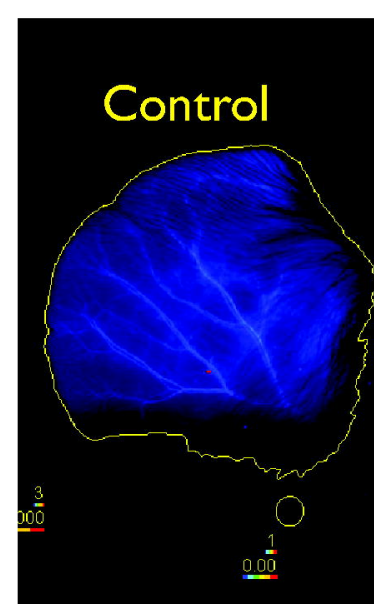

$+\operatorname{RvD2}$
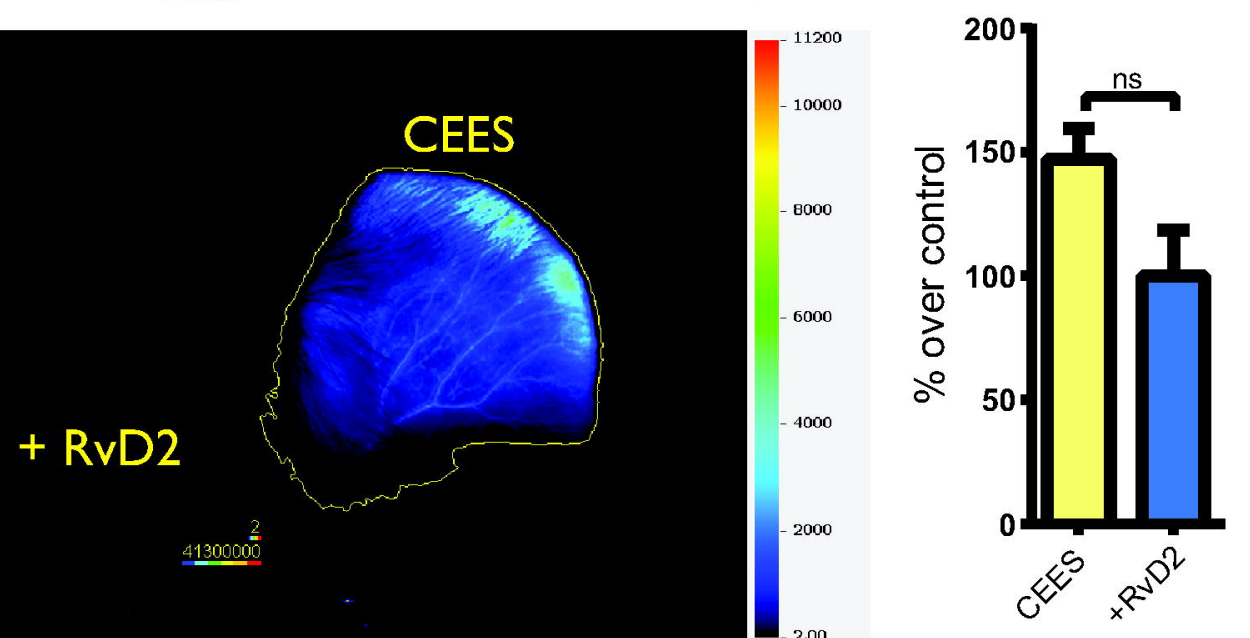

C
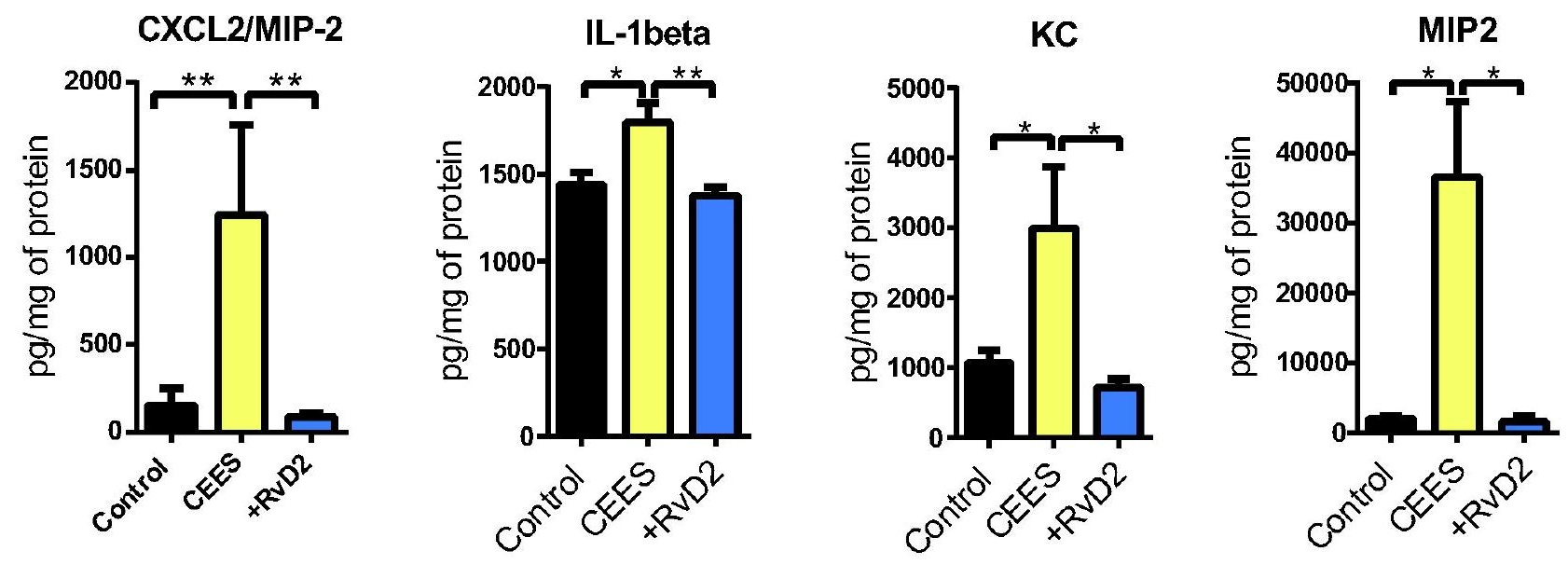
Figure 5

A

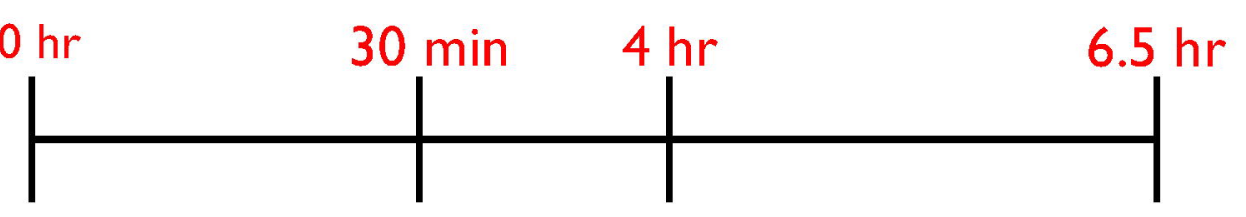
$\mathrm{R}: \mathrm{CS}$ tear gas
$(20 \mu \mathrm{L}, 200 \mathrm{mM})$
$\mathrm{L}:$ vehicle (DMSO)
Resolving agents

Sample collection

B

Ear thickness

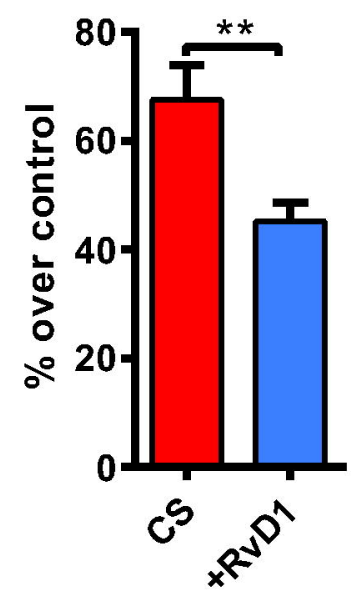

Ear punch biopsy weights

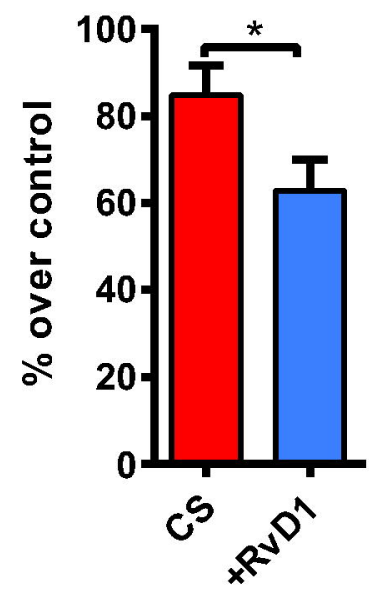

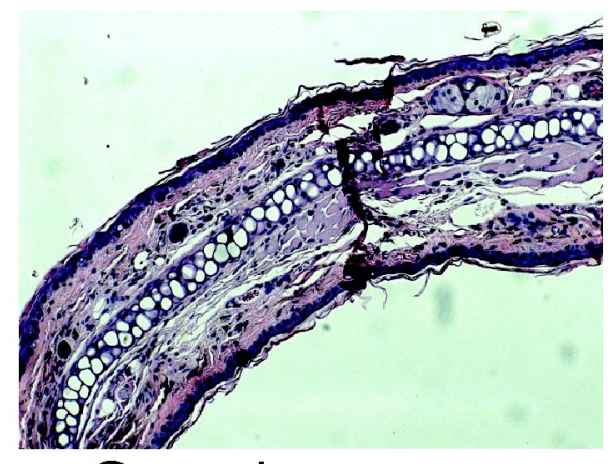

Control

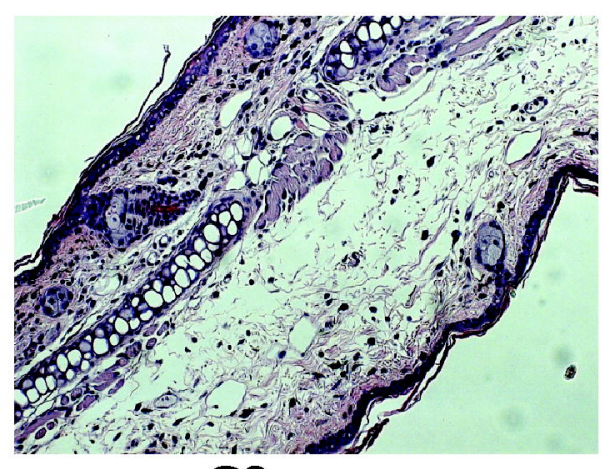

CS

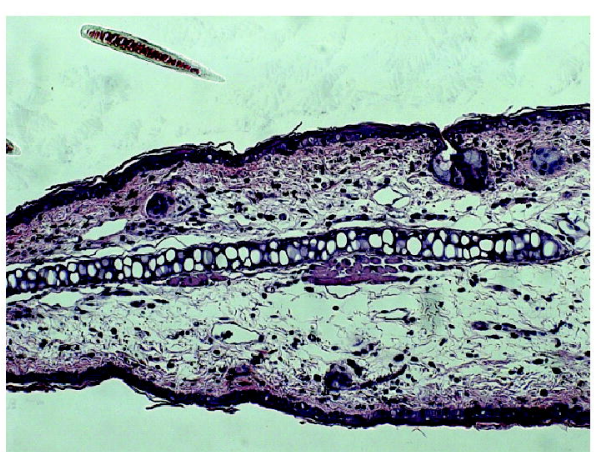

$C S+R v D I$
D

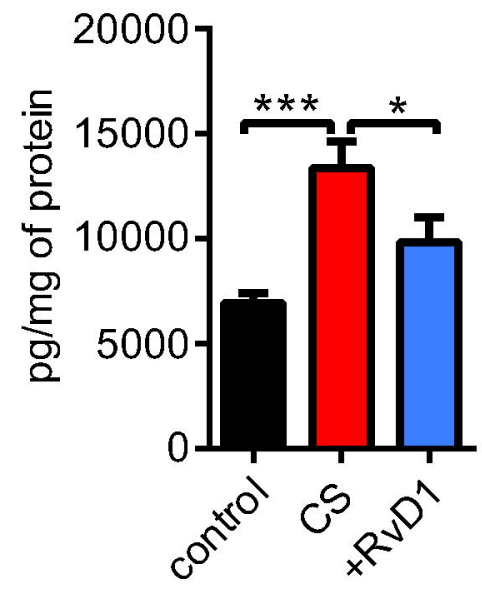

$\mathrm{KC} / \mathrm{CXCL1}$

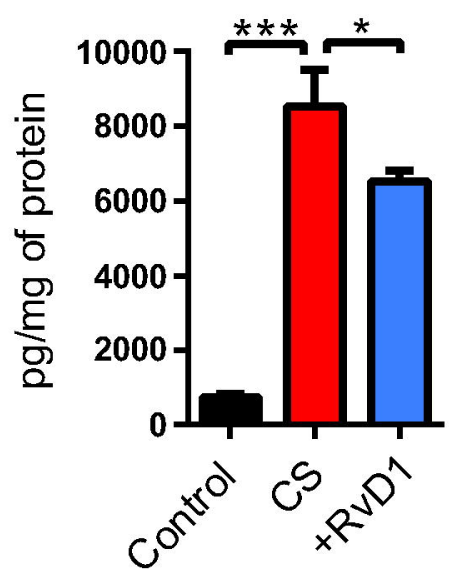

MMP-9

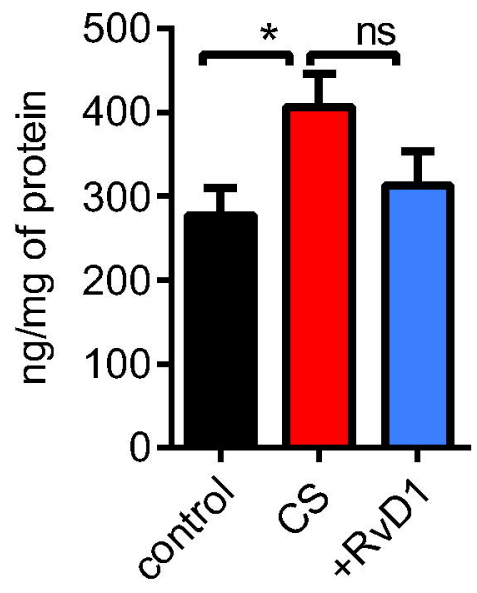


A

\section{Ear thickness}

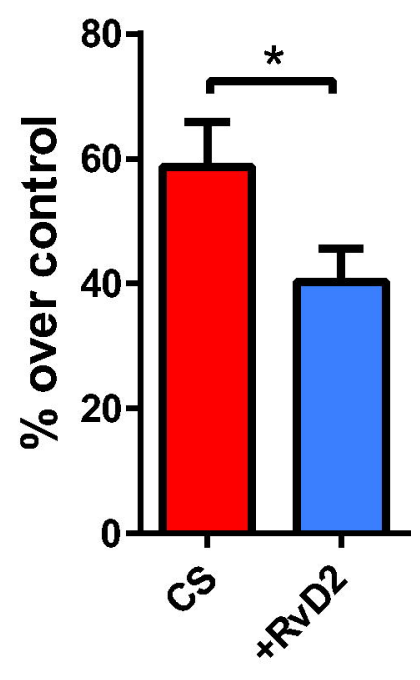

Ear punch biopsy weights

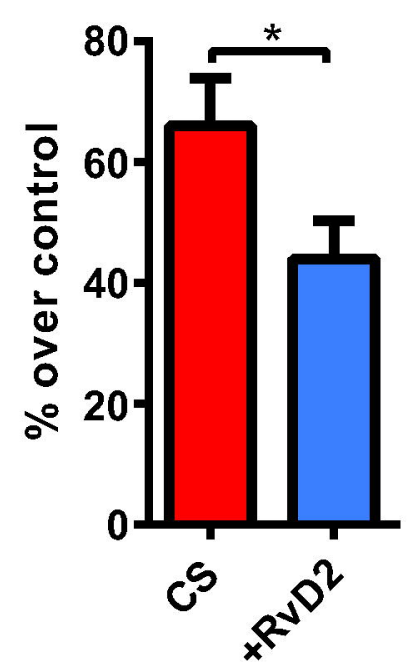

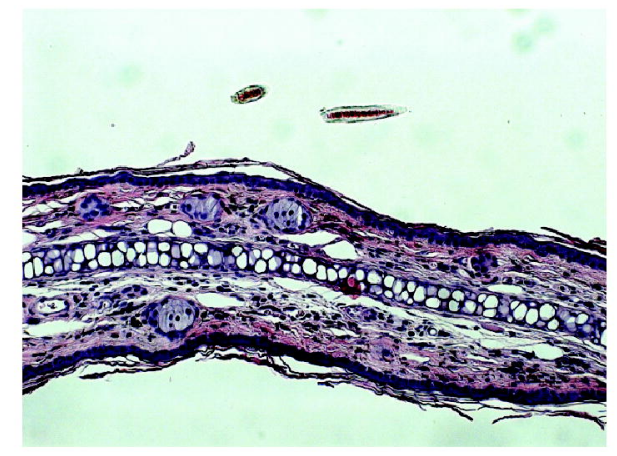

Control

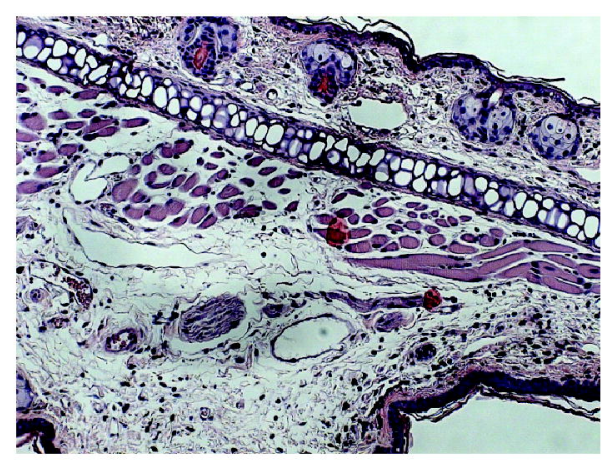

CS

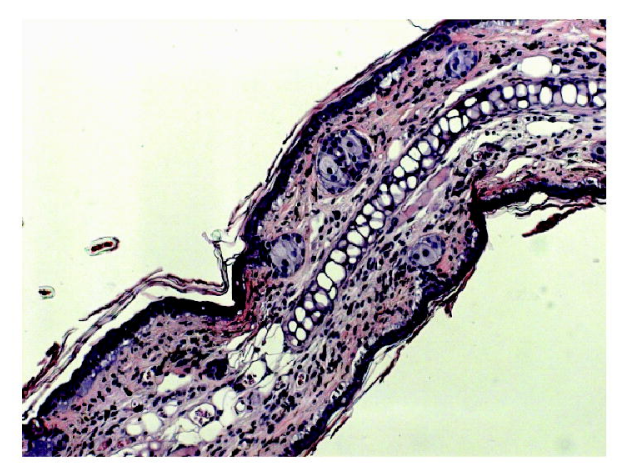

$\mathrm{CS}+\mathrm{RvD2}$
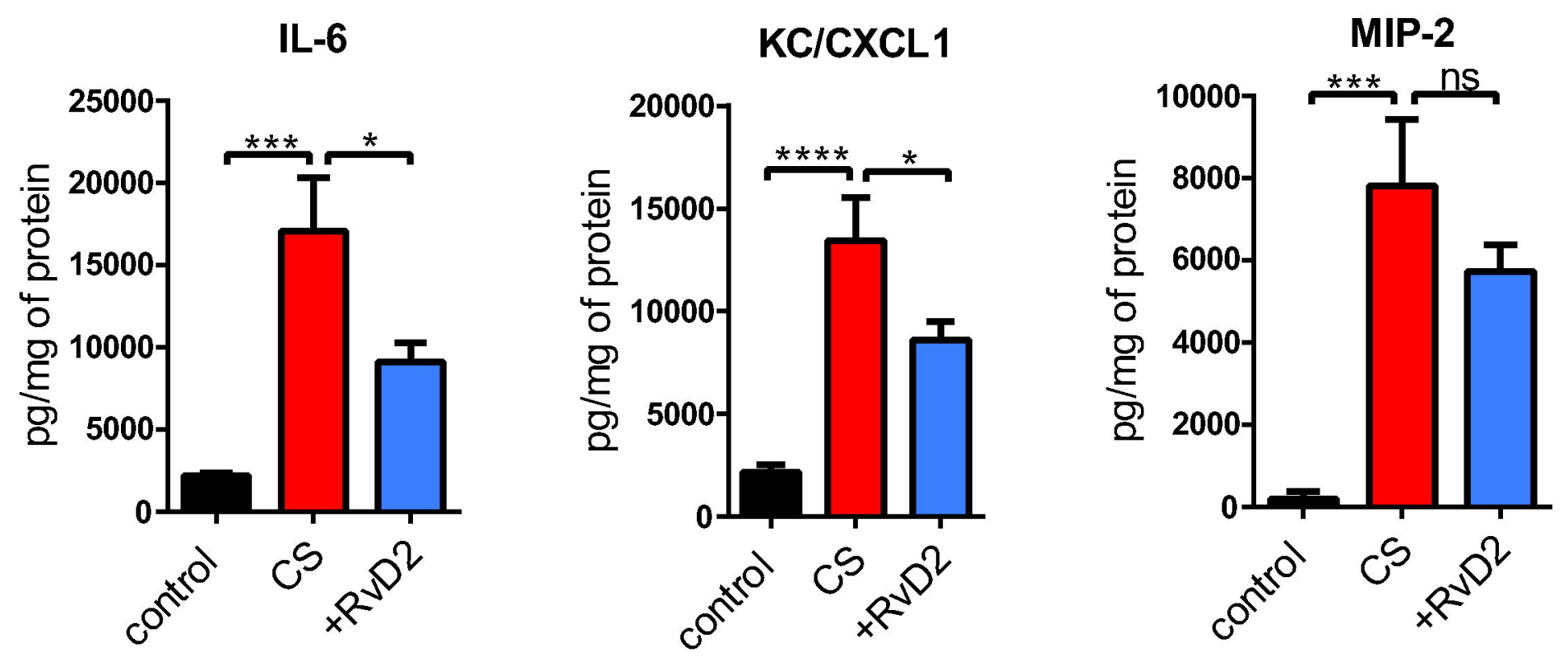
bioRxiv preprint doi: https://doi.org/10.1101/749184; this version posted August 29, 2019. The copyright holder for this preprint (which was not certified by peer review) is the author/funder, who has granted bioRxiv a license to display the preprint in perpetuity. It is made available under aCC-BY-NC-ND 4.0 International license.

\section{CEES or}

\section{CS tear gas}

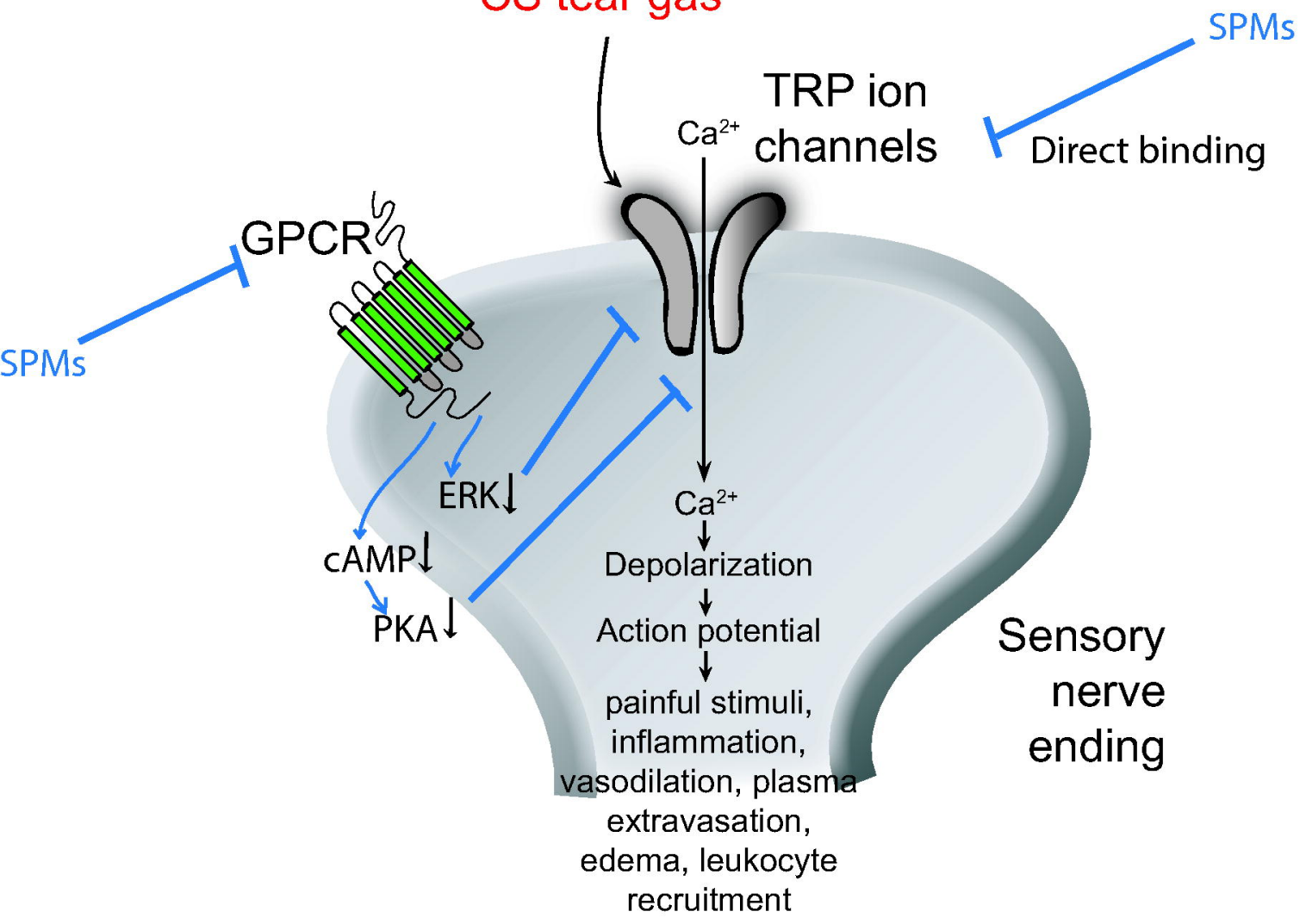

